\title{
TMB is linked with prognosis
}

Limited molecular markers are available to guide therapeutic decisions for patients with advanced-stage colorectal cancer (CRC). Now, a comprehensive mutational analysis of CRC samples indicates that several genetic features provide prognostic information in this setting.

This analysis was performed on a subset of tumour specimens from 2,326 patients with newly diagnosed, advanced-stage CRC collected before treatment in the CALGB/ SWOG 80405 trial. In this trial, patients received chemotherapy plus bevacizumab and/or cetuximab; no significant difference in overall survival (OS) was reported between arms. Mutational analysis of 11 genes was performed in 843 patients, 827 of which were profiled for microsatellite instability (MSI) status. Tumour mutational burden (TMB) was assessed in samples from 1,300 patients.
Longer OS durations were reported in patients with wildtype $B R A F$ versus mutated $B R A F$ (30.6 months versus 13.5 months; $P<0.001)$ or wildtype $R A S$ versus mutated $R A S$ (32.1 months versus 25.0 months; $P<0.001)$. No significant differences in OS were observed between treatment arms.

Patients with MSI-high (MSI-H) tumours receiving bevacizumab had longer median OS than those receiving cetuximab: 30.0 months versus 11.9 months $(P<0.001)$. "This finding has important implications for the treatment of this patient subgroup, for which immunotherapy is not approved yet in the first-line setting," says lead author

Federico Innocenti.

Among tumours evaluated for both TMB and microsatellite status, $89 \%$ had microsatellite stability (MSS) and a median TMB of 6 mutations per $\mathrm{Mb}, 6 \%$ had MSI-H (median TMB: 52 mutations per $\mathrm{Mb}$ ) and the remainder were classified as MSI-low (no TMB results available). In patients with tumours with MSS and high TMB ( $>8$ mutations per $\mathrm{Mb}$ ), median OS was longer than in those with tumours with MSS and low TMB (33.8 months versus 28.1 months; $P=0.02$ ). No significant differences in OS were observed between treatment arms. "We will now investigate if patients with high TMB are more responsive to immune-checkpoint inhibition in this setting," comments Innocenti.

"The results of these analyses and their future clinical testing could provide a new classification for precision medicine in CRC, improving the survival of patients with this disease," he concludes.

Diana Romero

ORIGINAL ARTICLE Innocenti, F. et al. Mutational analysis of patients with colorectal cancer in CALGB/SWOG 80405 identifies new roles of microsatellite instability and tumor mutational burden for patient outcome J. Clin. Oncol. https://doi.org/10.1200/JCO.18.01798 (2019)

\section{Brain metastases respond to neratinib plus capecitabine}

Brain metastasis from HER2-positive

breast cancer remains a major clinical challenge owing to the lack of approved treatments, particularly in the setting of central nervous system (CNS) recurrence after radiotherapy and/or surgery. Previous findings from cohort 1 of the phase II TBCRC 022 study demonstrated that the pan-HER tyrosine kinase inhibitor neratinib had only modest activity as monotherapy against brain metastases. Now, new data from TBCRC 022 cohorts $3 \mathrm{~A}$ and $3 \mathrm{~B}$ establish the efficacy of neratinib plus capecitabine against brain metastases from refractory, HER2-positive breast cancers.

The phase II single-arm studies enrolled women with lapatinib-naive (cohort 3A; $n=37$ ) or lapatinibpretreated (cohort 3B; $n=12$; closed early owing to slow accrual) disease with measurable, progressive, HER2-positive brain metastases, who received neratinib plus capecitabine during each 21-day cycle (14 days on, 7 days off). The primary end point was investigator-defined composite CNS

new data ... establish the efficacy of neratinib plus capecitabine against brain metastases from refractory, HER2-positive breast cancers objective response rate (ORR).

The cohort 3A composite CNS ORR was $49 \%$ (95\% Cl 32-66\%), whereas the cohort 3B CNS ORR was $33 \%(95 \% \mathrm{Cl}$ 10-65\%). In cohorts $3 \mathrm{~A}$ and $3 \mathrm{~B}$, median progression-free survival was 5.5 and 3.1 months, respectively, and median overall survival was 13.3 and 15.1 months, respectively.

No grade $\geq 4$ adverse events occurred. "Grade 2 and 3 diarrhoea was the most common side effect ( $29 \%$ in cohorts $3 \mathrm{~A}$ and $3 \mathrm{~B}$ combined) and we asked patients to take preventative medication for this when

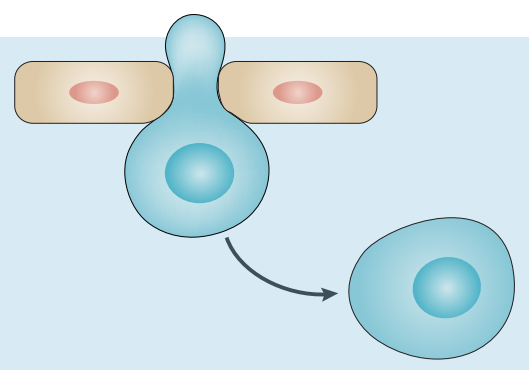

Credit: S. Bradbrook/Springer Nature Limited

they started the trial," explains lead investigator Rachel Freedman. The other most frequent grade 2 and 3 adverse events were nausea, vomiting and fatigue.

These findings suggest that the efficacy of neratinib is enhanced by chemotherapy in the setting of HER2-positive brain metastases. "Future studies are looking at other combinations in this setting, such as T-DM1-neratinib," concludes Freedman.

Conor A. Bradley

ORIGINAL ARTICLE Freedman, R. A. et al. TBCRC 022: a phase II trial of neratinib and capecitabine for patients with human epidermal growth factor receptor 2-positive breast cancer and brain metastases. J. Clin. Oncol. https://doi. org/10.1200/JCO.18.01511 (2019) 